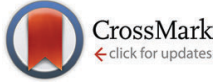

Cite this: Phys. Chem. Chem. Phys., 2016, 18, 7367

Received 1st January 2016, Accepted 4th February 2016

DOI: $10.1039 / c 6 c p 00012 f$

www.rsc.org/pccp

\title{
Amino-functionalized breath-figure cavities in polystyrene-alumina hybrid films: effect of particle concentration and dispersion $\dagger$
}

\author{
Lakshmi V., Annu Raju, Resmi V. G., Jerin K. Pancrecious, Rajan T. P. D.* and \\ Pavithran C.
}

\begin{abstract}
We report the formation of breath-figure (BF) patterns with amino-functionalized cavities in a BF incompatible polystyrene (PS) by incorporating functionalized alumina nanoparticles. The particles were amphiphilic-modified and the modifier ratio was regulated to achieve a specific hydrophobic/hydrophilic balance of the particles. The influence of the physical and chemical properties of the particles like particle concentration, the hydrophobic/ hydrophilic balance, etc., on particle dispersion in solvents having different polarity and the corresponding changes in the BF patterns have been studied. The amphiphilic-modified alumina particles could successfully assist the BF mechanism, generating uniform patterns in polystyrene films with the cavity walls decorated with the functionalized alumina particles, even from water-miscible solvents like THF. The possibility of fabricating free-standing micropatterned films by casting and drying the suspension under ambient conditions was also demonstrated. The present method opens up a simple route for producing functionalized BF cavities, which can be post-modified by a chemical route for various biological applications.
\end{abstract}

\section{Introduction}

Micropatterned polymer surfaces with functionalized cavities have been of great research interest during the past decade due to their potential use in several advanced applications. ${ }^{1-5}$ Indeed, the fabrication of materials having specific surface functionalities with a controlled distribution is a challenging field of research. Functionalized cavities can be conventionally obtained either by a multi-step approach often called the ex situ process $^{6,7}$ or by a single-step method called the in situ generation. ${ }^{8,9}$ The ex situ process involves post-modification of pre-fabricated non-functionalized cavities by different chemical reactions or by grafting techniques. However, the in situ generation process has been gaining more attention since it avoids the difficulty in the ex situ approach due to the involvement of a multi-step process and the complexity of chemical reactions.

The breath-figure (BF) technique, a facile dynamic templating method wherein self-organization of water droplets is exploited

Materials Science and Technology Division, CSIR-National Institute for Interdisciplinary Science and Technology (CSIR-NIIST), Council of Scientific and Industrial Research (CSIR), Thiruvananthapuram 695019, India.

E-mail: lekshmyv86@gmail.com, tpdrajan@rediffmail.com; Fax: +91-471-2491712; Tel: +91-471-2515327

$\dagger$ Electronic supplementary information (ESI) available: FTIR analysis of silane treated alumina and styrene modified alumina, optical transmission microscopy (OTM) images of patterned film, and TGA of amphiphilic-modified alumina particles. See DOI: 10.1039/c6cp00012f for phase separation in a polymer solution and formation of micropatterned films, offers a convenient, cost-saving and versatile procedure for in situ generation of functionalized micropatterned polymeric surfaces. ${ }^{10-12}$ Although the single step process of 'in situ' functionalization of polymeric patterns is a viable approach, the process is limited to the polymeric or amphiphilic structures which are compatible with BF formation. ${ }^{13,14}$ In addition, the functionalities were found in the rest of the film. The blend of polymers could achieve a specific distribution of its different components within or out of the cavity. ${ }^{15,16}$ Alternatively, functionality enrichment inside the cavity of a patterned polymer film was obtained by utilization of self-assembly of functionalized nanoparticles., ${ }^{3,17}$ They were generally prepared by dispersing or in situ generation of inorganic nanoparticles in a BF compatible polymer. It is well known that the condensed water droplets on the cool surface of the polymer solution arrange hexagonally by thermo-capillary and Marangoni force. ${ }^{11,18,19}$ These droplets are stabilized either by the precipitated polymer or the adsorbed inorganic particles. In particle-assisted stabilization, the adsorbed particles placed at the water droplet-solvent interfaces are further transported into the three-phase contact line under hydrodynamic drag force. $^{20}$ The complete evaporation of the solvent and water leaves BF cavities decorated with particles. However, the physical and chemical nature of the adsorbed particles influences the pore morphology. There have been several efforts to utilize nanoparticles as BF components to form hybrid structured films 
with cavities functionalized with particles. $^{20-28}$ Boker and co-workers $^{3}$ prepared patterned polystyrene films with BF cavities decorated with ligand-stabilized CdSe particles by the dispersion method. Nevertheless, CdSe particles do not assist the BF mechanism but assemble spontaneously at the interface for the selective functionalization of BF cavities. Sun et al. ${ }^{20,23}$ utilized Pickering emulsions along with the BF mechanism to achieve circular rings of hydrophilic silica particles on patterned films of linear PS. They studied the particle-assisted BF mechanism by varying the size, wettability and quantity of the particles.

In this article, we report the formation of BF patterns with amino-functionalized cavities from a BF incompatible PS by incorporating amphiphilic-modified alumina nanoparticles. Alumina particles were modified with a mixed solution of aminopropyltriethoxy silane (AS) and vinyl triethoxysilane (VS) followed by the 'in situ' polymerization with the styrene monomer through the vinyl moiety. Interestingly, we observed that the presence of amphiphilic alumina particles could stabilize the $\mathrm{BF}$ array on the PS solution by the fluid directed self-assembly process. Moreover, the physical and chemical properties of the particles also play a vital role in deciding the pore morphology. The influence of particle concentration and the hydrophilic/ hydrophobic ratio of the particles on the pore morphology of the BF patterns gave an insight into the particle-assisted BF mechanism operating in the present system. We succeeded in fabricating the patterned polystyrene alumina hybrid nanocomposite film with amino-functionalized alumina particles embedded inside the cavity wall. We also demonstrated the preparation of free-standing patterned films using the present system. The films can find several applications since the amino functionality can be easily modified with several substances by post-modification of the cavity through the reactive amino groups. $^{29-33}$

\section{Experimental section}

\subsection{Materials}

The materials used in this study were alumina powder (aps. $100 \mathrm{~nm}$, Sumitomo Corporation, Japan), polystyrene (GPPS, local source), 3-aminopropyltriethoxysilane (AS), vinyltriethoxysilane (VS), styrene (Aldrich Chemicals), benzoyl peroxide (S.D Fine Chem Ltd, India), tetrahydrofuran (THF), chloroform (CFM) and carbon disulphide (CS2) (Synthetic reagents, Merck Specialties Pvt. Ltd, India).

\subsection{Methods}

Amphiphilic-modified alumina particles were prepared by treating the particles with hydrolyzed solution of a mixture of 3-aminopropyltriethoxysilane (AS) and vinyl triethoxysilane (VS) (3 wt\% of alumina), followed by the in situ polymerization of styrene through the vinyl group. Styrene/alumina molar ratios were varied from 0.05 to 0.1 . The AS/VS mixture of equimolar ratio in the ethanol-water mixture ( $95: 5 \mathrm{v} / \mathrm{v})(1 \mathrm{wt} \%$ solution) was magnetically stirred for 30 minutes. The hydrolyzed silane solution thus obtained was added to a $10 \mathrm{wt} \%$ alumina/ethanol-water suspension, which was pre-dispersed by sonication (Branson 3510 Sonicator, $100 \mathrm{~W}-40 \mathrm{~Hz}$ ). The above mixture was then magnetically stirred for $24 \mathrm{~h}$. The silane-modified particles were separated by centrifugation, washed with the ethanol-water mixture and dried at $60{ }^{\circ} \mathrm{C}$.

Polymer modification was carried out by dispersing the silane modified particles in toluene containing a desired amount of styrene and benzoyl peroxide ( $0.5 \mathrm{wt} \%$ of styrene). Polymerization was initiated in a nitrogen atmosphere at 70-80 ${ }^{\circ} \mathrm{C}$ for $10-12 \mathrm{~h}$ while stirring the contents magnetically. The styrene-modified amphiphilic particles were coagulated by adding methanol and separated by filtration. The residue was washed with the methanol-toluene mixture to remove the free polymer and dried at $100{ }^{\circ} \mathrm{C}$ in an air oven.

Polystyrene-alumina (PSA) suspensions for micro-patterned composite films were prepared by dispersing the modified amphiphilic alumina particles in tetrahydrofuran (THF), chloroform (CFM) and carbon disulphide (CS2) solutions of PS. The amount of particles was varied from 0 to $4 \mathrm{wt} \%$ of polystyrene. The patterned films were prepared as follows. The PSA solution was mixed ultrasonically and drop cast $(3 \mu \mathrm{L})$ on a glass slide within 2 minutes. It was then dried for solvent evaporation at ambient temperatures and humidity, followed by drying at $60{ }^{\circ} \mathrm{C}$.

The amphiphilic-modified alumina particles were characterized by FTIR (Perkin Elmer Series FTIR Spectrometer-2), the organic content of styrene-modified alumina particles was determined using a thermo-gravimetric analyzer (TGA, Hitachi High-Tech STA 7200) and surfaces of the drop-cast residues were characterized by SEM (JEOL JSM-5600LV) using samples which were gold-coated by employing a JEOL JFC-1200 fine coater. Composite films with nonpatterned surfaces were characterized by XRD (Xeuss SAXS/WAXS system by Xenocs) and water contact angle measurements were performed using a Sigma 701 Tensiometer.

\section{Results and discussion}

Alumina particles used were nearly spherical in shape and showed strong crystalline peaks in XRD (ESI $\dagger$ ). Polystyrene (PS) showed a molecular weight of $\sim 3.2 \times 10^{5}$, a polydispersity of $\sim 1.6$, a water contact angle of $101^{\circ}$ and a broad peak at $2 \theta \approx$ $20^{\circ}$ in XRD due to its highly amorphous linear structure. Alumina particles treated with a hydrolyzed AS/VS mixture exhibited bifunctionality as a result of the coating of particles with inter-condensation products of AS and VS. FTIR spectra confirm the introduction of these functionalities by the absorption bands at 2925, 2855 and $1524 \mathrm{~cm}^{-1}$ for the amino propyl group, at $1641 \mathrm{~cm}^{-1}$ for the vinyl group and at $1130 \mathrm{~cm}^{-1}$ for $\mathrm{Si}-\mathrm{O}-\mathrm{Si}$ stretching (ESI $\dagger)$. The lyophilic vinyl group was expected to promote the dispersion of the particles in PS solutions. However, its suspensions in THF, CFM and CS2 were highly unstable. The stability of the suspensions was improved by styrene modification of the particles which occurred through vinyl groups as confirmed by the absence of the bands due to the vinyl group in FTIR. Suspensions stable for more than $30 \mathrm{~min}$. were used for film preparation. They were obtained by using a styrene to alumina molar ratio of 0.07 and above. 
It should be noted that the styrene-modified particles (SA) with a styrene to alumina molar ratio of $0.07,0.09$ and 0.1 were used in the present study. The hydrophobic/hydrophilic ratio of these particles was $\sim 2,2.6$ and 4, respectively. (We calculated the hydrophobic/hydrophilic ratio of the particles from TGA analysis on the assumption that the AS and VS condenses on the particles in an equimolar ratio (ESI $\dagger)$ ). In the following text the composites of these particles are referred to as $\mathrm{P} x(y \mathrm{SA})$ where 'P' stands for polystyrene, 'SA' for styrene-modified alumina, ' $y$ ' for the hydrophobic/hydrophilic ratio of SA particles and ' $x$ ' denotes the percentage of ySA particles in the composite.

The effect of particle concentration on its dispersion in PS was studied by XRD analysis of Px(2SA) composite films with non-patterned surfaces (Fig. 1). Whereas alumina particles exhibited intense peaks at $2 \theta=25.7^{\circ}$ and $35.3^{\circ}$ (plot A) due to particle-particle interaction, PS was XRD silent at $2 \theta$ between $24^{\circ}$ and $36^{\circ}$ (plot B). In the composites, the peaks due to alumina were weak up to a particle concentration of $3 \mathrm{wt} . \%$ (plots $\mathrm{C}$ and $\mathrm{D}$ ) and then the intensity appreciably increased upon increasing the particle concentration to $4 \mathrm{wt} \%$ and $5 \mathrm{wt} \%$ (plots $\mathrm{E}$ and F). Composites with well dispersed crystalline nanoparticles generally show shallow and broad peaks for the particles and the peaks become dominant upon particle agglomeration. ${ }^{34}$ The present results indicated that the size of the particles and particleagglomerates increased due to further agglomeration when particle loading exceeded $3 \mathrm{wt} \%$ of polystyrene.

The film from solution of neat PS in THF $\left(15 \mathrm{mg} \mathrm{mL}^{-1}\right)$ produced by drop casting and drying on a glass plate under ambient conditions did not show a BF pattern in accordance with the previous results (ESI $\dagger$ ). ${ }^{35}$ Addition of a desired amount of the modified alumina particles to the above solution produced regular BF patterns on the film. Fig. 2 shows variation in SEM images of $\mathrm{P} x(2 \mathrm{SA})$ films from THF upon varying the particle content

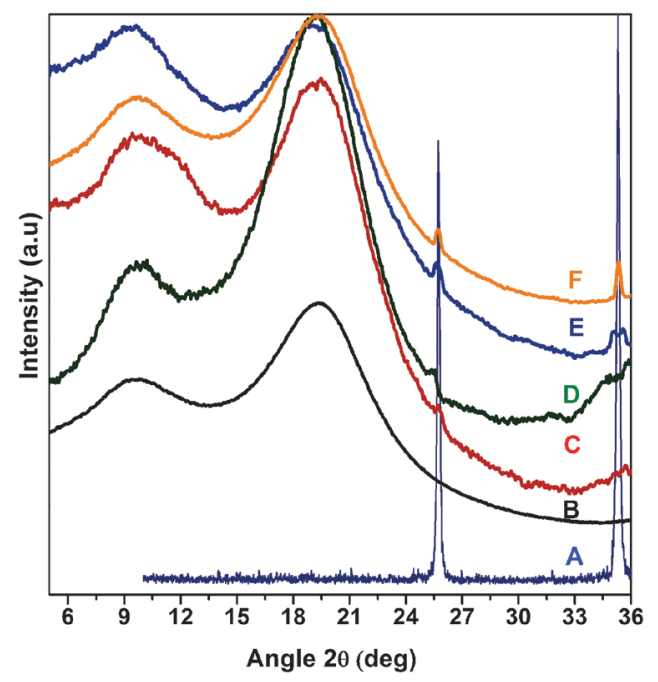

Fig. $1 \mathrm{XRD}$ at $2 \theta$ between $5^{\circ}$ and $36^{\circ}$ of polystyrene/modified-alumina composite $[\mathrm{Px}(2 \mathrm{SA})]$ films in comparison with that of the polystyrene matrix and alumina particles, showing the effect of particle concentration on its dispersion in the polymer matrix. (A) alumina, (B) polystyrene, (C) P2(2SA), (D) P3(2SA), (E) P4(2SA), and (F) P5(2SA).
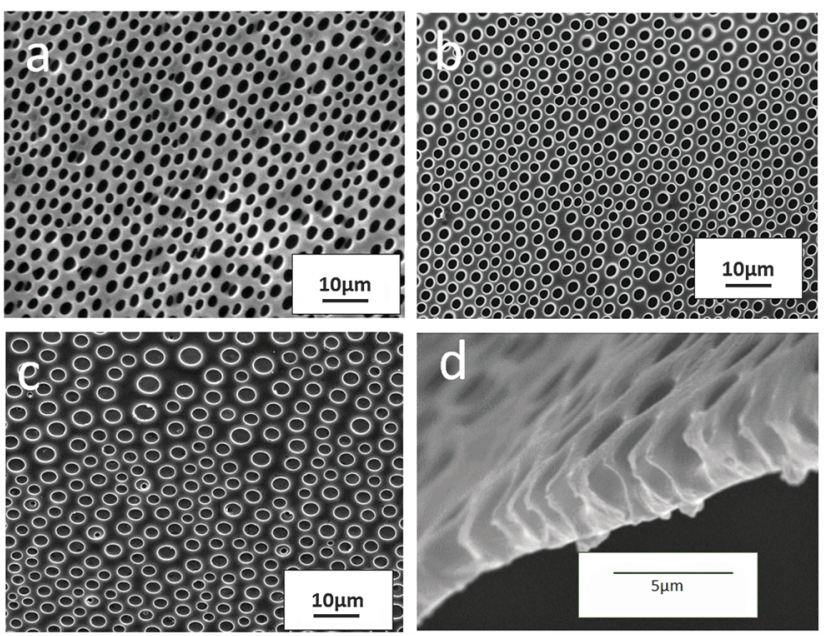

Fig. 2 SEM images of patterned composite films (a) P2(2SA), (b) P3(2SA), (c) P4(2SA) and (d) cross-section of P3(2SA) film from THF, showing the effect of particle concentration on pore morphology of the BF pattern.

in the range of 2-4\%. P2(2SA) showed irregular arrays of concavities while the microstructure became relatively uniform in $\mathrm{P} 3(2 \mathrm{SA})$ with concavities having a diameter close to that observed for the P2(2SA) film. A further increase in the particle concentration (P4(2SA)) resulted in a non-uniform microstructure with concavities having a wide range of diameters (Fig. 2c). More specifically, the morphology of the patterns in the composite film was dependent on the particle concentration such that particle concentration around $3 \mathrm{wt} \%$ was critical to form the most closely packed arrays of concavities with a diameter in a narrow range.

So as to understand the BF mechanism in the systems, we analyzed Px(2SA) composite films with plain surfaces which were obtained by casting and drying the suspensions in THF $\left(15 \mathrm{mg} \mathrm{mL} \mathrm{m}^{-1}\right)$ in a glove box. Water contact angle measurements on the films showed that the angle gradually decreased with increasing particle concentration. The angle gradually decreases from $101^{\circ}$ for PS to $94^{\circ}$ for P2(2SA), $87^{\circ}$ for P3(2SA), $82^{\circ}$ for $\mathrm{P} 4(2 \mathrm{SA})$ and to $76^{\circ}$ for P5(2SA). Hydrophilic properties of the composite surfaces increased with increasing particle concentration. Such a trend can be expected when the surface layer of the films carry an increasing amount of particles, exposing the hydrophilic amino groups at the surfaces. This was confirmed by treating the film with ninhydrin, which produced blue colored spots upon reacting with amino groups (ESI $\dagger$ ).

So far, several discussions on the particle assisted $\mathrm{BF}$ mechanism have been reported..$^{21-25}$ The ability of nanoparticles to assemble at the water-droplet/suspension interface by hydrodynamic drag force is well established. It should be noted that the BF stabilization by particles depends on the nature of the particles, the matrix polymer, the environmental conditions and the solvent used. In general, fast evaporation of the solvent from PSA suspension follows reduction in volume/thickness of the film at a rate higher than that of the particle settling rate. As a result, particles begin to appear in the surface layer even at the early stages of drying. Once the water droplet condenses on the surface of the suspension, the particles are adsorbed at the 
droplet-suspension interfaces. The stabilization of the water droplet occurred due to the adsorbed particles at the interfaces, which act as a mechanical barrier to coalescence. Fine dispersion of the particles upsurge the surface coverage and number density of the particles, while large agglomerates tend to settle down and the condition to be adsorbed at the interface is offset. The size of the water droplet indeed depends on the stabilization capability of the adsorbed particles. The stabilized droplets, along with the adsorbed particles, form BF patterns by hydrodynamic drag force and Marangoni force. Drying of water and the remaining solvent leaves concavities functionalized with particles.

In the case of P2(2SA), the number of particles in the suspension was insufficient to stabilize the water droplets in a uniform manner to form regular hexagonal arrays. Practically, this was possible only by dispersing a maximum amount of particles in the suspension, as for P3(2SA). Any deviation from this resulted in a non-uniform pattern. Above the dispersion loading limit, particle agglomeration reduced the effective number of particle domains in the suspension. Apart from that, the large agglomerates were not easily carried with the water droplets towards the three phase contact line. This leads to irregular patterns with varying cavity size in P4(2SA).

Incompatibility of neat PS in THF to BF formation was due to the miscibility of THF with water droplets. ${ }^{36}$ The system became BF compatible when particles were dispersed in it. The particles assembled at the diffusion layer of THF and water acted as a barrier to further dissolution, stabilizing the droplets. It should be noted that no particles are seen at the edge of the patterns, as they are preferably located at the interior of the cavity wall. Since the particles employed for the BF stabilization mechanism were partially hydrophobic-modified, they were partially immersed in the organic phase. Moreover, the high interfacial tension between the polymer and the water droplet forces the polymer precipitation around the droplet which creates a roof over every pore, which mask the underneath particles. ${ }^{23}$ The SEM image of the cross-section of P3(2SA) film (Fig. 2d) showed such a roof, covering the larger interior cavity with a smaller cavity opening.

Detection of amino functionalization inside the BF cavity was carried out by treating the BF patterned film with fluorescamine which is not florescent by itself but forms a florescent adduct upon reacting with primary amine. ${ }^{8}$ Fig. 3 shows the fluorescence microscopy image of the P3(2SA) micropatterned film after treatment. The cavities were functionalized with amino groups of the particles which also proves that the particles segregate at the water droplet/PS interface during BF formation.

The influence of the hydrophobic/hydrophilic balance of the amphiphilic-modified alumina particles on the pore morphology of BF patterns from THF was also investigated. Fig. 4 shows the SEM images of the patterns for P3(2SA), P3(2.6SA) and P3(4SA). We can see that 2.6SA particles help to achieve a better pattern than 2SA and 4SA particles, although the average pore diameter measured from the patterns was in a close range of 1.56-1.66 $\mu \mathrm{m}$. On the other hand, the feature density increased from $14 \times 10^{7} \mathrm{~cm}^{-2}$ for P3(2SA) to $15 \times 10^{7} \mathrm{~cm}^{-2}$ for P3(2.6SA), while P3(4SA) exhibited scattered morphology with a very low feature density of $6 \times 10^{7} \mathrm{~cm}^{-2}$. To be specific, the hydrophobic/hydrophilic



Fig. 3 Fluorescence microscopy image of micropatterned P3(2SA) film from THF after treatment with fluorescamine.

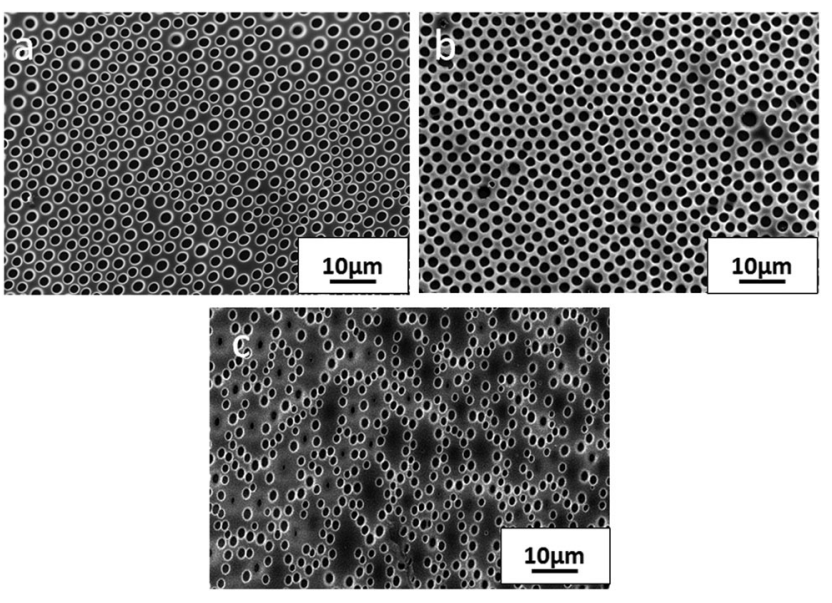

Fig. 4 SEM image of micropatterned composite films (a) P3(2SA), (b) P3(2.6SA) and (c) P3(4SA) from THF, showing the effect of hydrophobicity of alumina particles on BF pore morphology.

balance of the particles played a significant role in determining the morphology of BF patterns.

In order to explain the observed morphological variation, dispersion of the particles in PS/THF solution and suspension stability with increasing hydrophobicity of the particle have to be considered. Obviously, increased hydrophobicity improves dispersion of the particles in PS/THF solution thereby enhancing the number advantage of particles towards the water dropletsolvent interface. So the regularity and pore density increased with increased hydrophobicity of 2.6SA particles when compared to that of 2SA particles. The water miscible THF solvate the amino moiety and an electro-steric stabilization is likely to happen in these circumstances which promote the dispersion even at a low hydrophobic/hydrophilic ratio of $2: 1$. A further increase in the organic chain length as in 2.6SA particles increases the dispersity of particles indicated by an increase in the feature density of P3(2.6SA) drop cast film. Nevertheless, we are observing a defective pattern for P3(4SA) in contrast to an increase in the uniformity. As the hydrophobic ratio increases to $4: 1$, although effective dispersion was expected, the stabilization capability of particles at the BF interface is reduced. This might be due to the fact that enhanced interaction between 4SA particles and the PS/THF solution is expected due to the increased chain length, so the highly hydrophobic 4SA particles reside more on the solvent phase in the diffusion layer of THF 
and condensed water droplets. This resulted in diffusion layer enlargement leading to an irregular pattern. This made us to conclude that there should be a balance between the hydrophilic to hydrophobic ratio for the particles to stabilize the water droplets effectively in polar solvents like THF. Sun et $a l^{23}$ reported that by increasing the quantity of alcohol in a suspension of silica particles, the regularity of BF patterns in PS from chloroform altered due to an increased amount of particles at the interface. This was attributed to alcohol, which served as a 'solvent surfactant'. In the present case, the amphiphilic-modified alumina particle itself behaves as a 'particle surfactant' and the adsorption at the BF interface depends on various factors like size, shape, concentration and wettability of the particle. When amphiphilic particles are used, the change in interactions of the particles with the polymer upon varying the hydrophobic/hydrophilic balance also should be considered for explaining the variations in BF patterns.

P3(ySA) from chloroform followed a different trend from that observed for the films from THF. Fig. 5 shows the SEM images of P3(2SA), P3(2.6SA) and P3(4SA) films. In contrast to their counterparts from THF, the pore size decreased, uniformity and feature density of BF patterns increased with the organic chain length. P3(2SA) showed an irregular pattern with an average pore size of $3.3 \mu \mathrm{m}$, while the average pore size decreased to $1.7 \mu \mathrm{m}$ for $\mathrm{P} 3(2.6 \mathrm{SA})$ and $1.5 \mu \mathrm{m}$ for $\mathrm{P} 3(4 \mathrm{SA})$ composite films.

It should be noted that the pore interval decreased with the hydrophobicity of particles, which remarkably increases the feature density. The hydrophilic amino moiety which is incompatible with the non-polar solvent always promote the capillary movement of particles towards the BF interface. The water immiscible nature of chloroform creates a sharp boundary at the interface where the particles align with its hydrophilic amino part into the water phase and the polymeric chain in the solvent phase. Highly irregular patterns with large cavities were formed on the hybrid film with 2SA particles in CFM in contrast to its analogy in THF. This could be due to the less achievement of effective dispersion of modified particles with a hydrophobic/hydrophilic
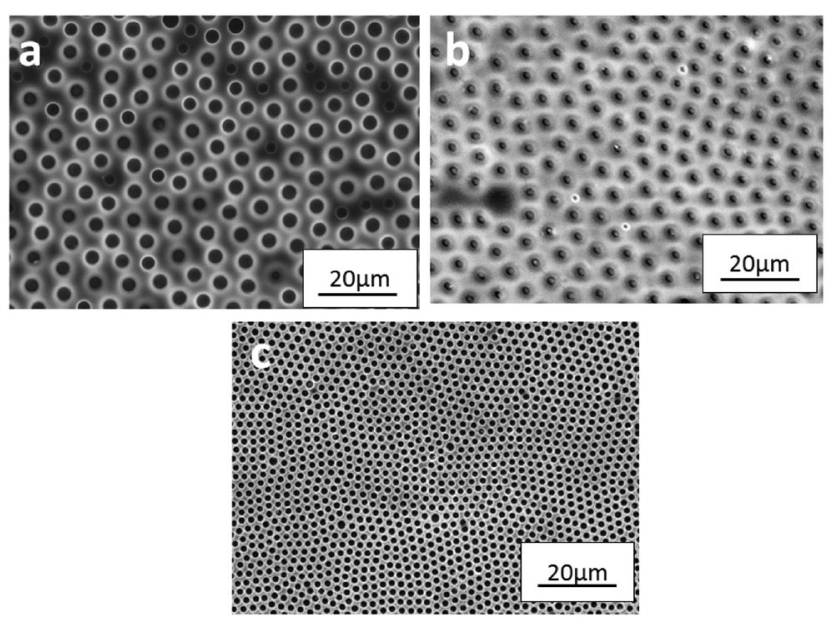

Fig. 5 SEM images of micropatterned composite films (a) P3(2SA), (b) $\mathrm{P} 3(2.6 \mathrm{SA})$ and (c) P3(4SA) from CFM, showing the effect of hydrophobicity of alumina particles. ratio of $2: 1$ in PS/CFM solution. Dispersion of the amphiphilicparticles occurs by steric repulsion from PS segments bound to the particles. Obviously, improvement in dispersion with increase in hydrophobicity and lack of electrostatic interaction between the solvent and the particles resulted in desired results for the P3(4SA) system. Moreover, the number of particles stabilizing the water droplets increases and the pore size decreases accordingly. This was confirmed by using another non-polar solvent carbon disulphide (CS2) for the preparation of BF patterned films. Fig. 6 shows the SEM images of drop cast film of composites P3(2SA), P3(2.6SA) and P3(4SA) from $\mathrm{CS}_{2}$. As expected the change in the pattern followed a similar trend as observed for the film from CFM. Hence, it is concluded that in nonpolar solvents, the amphiphilic nature of the particles is important but an increase in the hydrophobicity of the particles increases the uniformity and pore density until a maximum dispersion limit and further enhancement in hydrophobicity did not show appreciable refinement in the microstructure.

The pore size and feature density measured from the images of the BF pattern using imageJ are summarized in Table 1. It can be seen that the most uniform patterns, i.e. P3(2.6SA) from THF and P3(4SA) from CFM and CS2, exhibited nearly identical pore size. Generally, cavity size depends on the equilibrium size of the water droplets which in turn depends on the concentration of the solution, evaporation rate of the solvent, relative humidity and the stabilization capability of the particles. ${ }^{37}$ A slightly higher cavity size for the film from THF can be attributed to its miscibility with water droplets. Low miscibility with water and fast evaporation of non-polar solvents generally lead to concavities having a smaller diameter and lower pore density. The identical cavity size in the present case can be due to the achievement of a desired level of stabilization capability of the particles and their dispersion in the solvents to the same extent by particle surface modification.

Fig. 7 shows the photograph of micropatterned film of $3.0 \mathrm{~cm}$ diameter by casting the composite suspension in CFM in a Petri-dish and drying under ambient conditions, proving

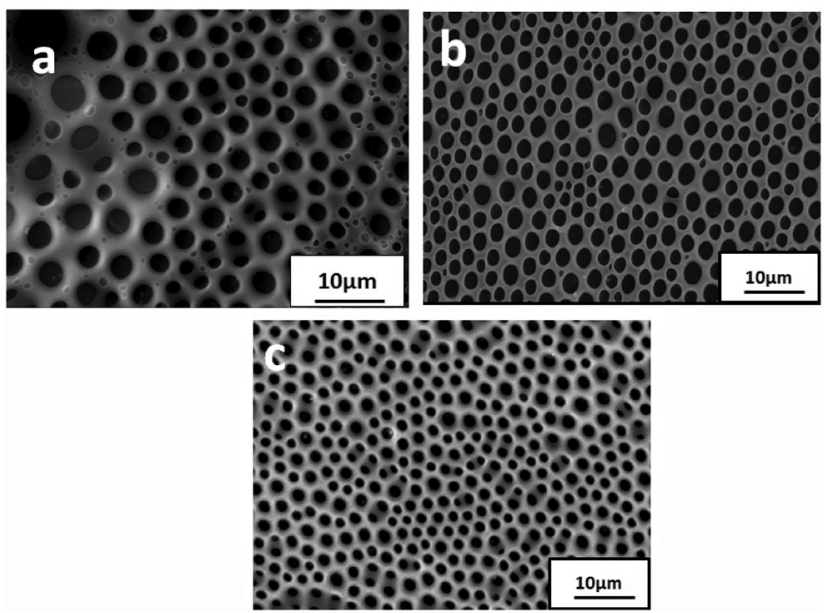

Fig. 6 SEM images of micropatterned composite films (a) P3(2SA), (b) P3(2.6SA) and (c) P3(4SA) from CS2, showing the hydrophobicity of alumina particles. 
Table 1 Effect of particle hydrophobicity in the cavity size and feature density of P3(2SA), P3(2.6SA) and P3(4SA) composite films from THF, CFM and CS2

\begin{tabular}{llll}
\hline Composite & Solvent & Cavity size $(\mu \mathrm{m})$ & $\begin{array}{l}\text { Feature density } \\
\text { (cavity per } \mathrm{cm}^{2} \text { ) }\end{array}$ \\
\hline P3(2SA) & THF & $1.66 \pm 0.08$ & $14 \times 10^{7}$ \\
P3(2.6SA) & & $1.61 \pm 0.09$ & $15 \times 10^{7}$ \\
P3(4SA) & & $1.56 \pm 0.26$ & $6.1 \times 10^{7}$ \\
& & $3.30 \pm 0.14$ & $3.0 \times 10^{7}$ \\
P3(2SA) & CFM & $1.70 \pm 0.06$ & $5.0 \times 10^{7}$ \\
P3(2.6SA) & & $1.50 \pm 0.04$ & $16 \times 10^{7}$ \\
P3(4SA) & & $2.9 \pm 1.04$ & $4.1 \times 10^{6}$ \\
P3(2SA) & CS2 & $2.2 \pm 0.37$ & $1.2 \times 10^{7}$ \\
P3(2.6SA) & & $1.5 \pm 0.21$ & $1.5 \times 10^{7}$ \\
P3(4SA) & & & \\
\hline
\end{tabular}



Fig. 7 Photograph of free-standing micropatterned P3(4SA) film from CFM along with the optical microscopic image.

that the present method also offers a facile route for the preparation of free-standing large size micropatterned films. Increased rigidity due to the inorganic phase ${ }^{38,39}$ could be an added advantage of the present system. Although the present work is carried out on a glass substrate, which facilitates the easy removal of the patterned film, we also investigated the effect of the substrate by drop casting the composite solutions of THF and CFM on a polypropylene (PP) film. PSA/CFM solution could achieve a regular BF array with an average cavity size of $2.4 \mu \mathrm{m}$ in the PP film. PSA/ THF solution does not produce any regular pattern. Further investigations are going on with the PP film.

\section{Conclusions}

Micropatterned polystyrene-alumina nanocomposite films with two-dimensional arrays of amino-functionalized concavities have been prepared from suspensions of amphiphilic-modified alumina particles in polystyrene solutions by the particle-assisted breath figure method. The influence of particle concentration and the hydrophobic/hydrophilic balance of the amphiphilic-modified alumina particles on the BF pattern from polar and nonpolar solvents was studied. A relatively uniform microstructure was obtained at maximum particle dispersion-loading and using particles having a different hydrophobic/hydrophilic balance for polar and non-polar solvents. The method offers a facile route for fabricating free-standing large-size micropatterned nanocomposite films with amino-functionalized concavities.
The applicability of the present investigation lies in the amino functionality inside the cavity, which can be post-modified for various biological and catalytic applications.

\section{Acknowledgements}

The authors thank CSIR, New Delhi, for the research fellowship and the Director and members of Materials Science and Technology Division, CSIR-NIIST. The authors are also thankful to Mr M. R. Chandran. Mrs Lucy Paul, Mrs Sowmya, Dr Boje Gowd, NIIST, respectively, for SEM and XRD analysis.

\section{References}

1 J. A. Hubbell and R. Langer, Tissue Eng., Chem. Eng. News, 1995, 73, 42-54.

2 L.-S. Wan, Q.-L. Li, P.-C. Chen and Z.-K. Xu, Patterned biocatalytic films via one-step self-assembly, Chem. Commun., 2012, 48, 4417-4419.

3 A. Böker, Y. Lin, K. Chiapperini, R. Horowitz, M. Thompson, V. Carreon, T. Xu, C. Abetz, H. Skaff, A. D. Dinsmore, T. Emrick and T. P. Russell, Hierarchical nanoparticle assemblies formed by decorating breath figures, Nature, 2004, 3, 302-306.

4 X. Xu, L. Heng, X. Zhao, J. Ma, L. Lin and L. Jiang, Multiscale bio-inspired honeycomb structure materials with high mechanical strength and low density, J. Mater. Chem., 2012, 22, 10883-10888.

5 A. Munoz-Bonilla and M. Fernández-García, Towards hierarchically ordered functional porous polymeric surfaces prepared by the breath figures approach, Prog. Polym. Sci., 2014, 39, 510-514.

6 J. Nishida, K. Nishikawa, S.-I. Nishimura, S. Wada, T. Karino, T. Nishikawa, K. Ijiro and M. Shimomura, Preparation of self-organized micropatterned polymer films having cell adhesive ligands, Polym. J., 2002, 34, 166-174.

7 D. Nyström, E. Malmström, A. Hult, I. Blakey, C. Boyer, T. P. Davis and M. R. Whittaker, Biomimetic surface modification of honeycomb films via a "grafting from" approach, Langmuir, 2010, 26, 12748-12754.

8 F. Galeotti, V. Calabrese, M. Cavazzini, S. Quici, C. Poleunis, S. Yunus and A. Bolognesi, Self-functionalizing polymer film surfaces assisted by specific polystyrene end-tagging, Chem. Mater., 2010, 221, 2764-2769.

9 S. Samanta, D. P. Chatterjee, R. K. Layek and A. K. Nandi, Multifunctional porous poly(vinylidene fluoride)-graftpoly(butyl methacrylate) with good $\mathrm{Li}+$ ion conductivity, Macromol. Chem. Phys., 2011, 212, 134-149.

10 L. Rayleigh, Breath Figures, Nature, 1911, 86, 416-417.

11 U. H. F. Bunz, Breath Figures as a Dynamic Templating Method for Polymers and Nanomaterials, Adv. Mater., 2006, 18, 973-989.

12 S. Yunus, A. Delcorte, C. Poleunis, P. Bertrand, A. Bolognesi and C. Botta, A route to self-organized honeycomb microstructured polystyrene films and their chemical characterization by ToFSIMS imaging, Adv. Funct. Mater., 2007, 17, 1079-1084. 
13 L. Billon, M. Manguian, V. Pellerin, M. Joubert, O. Eterradossi and H. Garay, Tailoring highly ordered honeycomb films based on ionomer macromolecules by the bottom-up approach, Macromolecules, 2009, 42, 345-356.

14 L. Billon, M. Manguian, V. Pellerin, M. Joubert, O. Eterradossi and H. Garay, Tailoring highly ordered honeycomb films based on ionomer macromolecules by the bottom-up approach, Macromolecules, 2009, 42, 345-356.

15 N. Park, M. Seo and S. Y. Kim, Particle and breath figure formation of triblock copolymers having self-complementary hydrogen-bonding units, J. Polym. Sci., Part A: Polym. Chem., 2012, 50, 4408-4414.

16 A. E. Saunders, J. L. Dickson, P. S. Shah, M. Y. Lee, K. T. Lim, K. P. Johnston and B. A. Korgel, Breath figure templated self-assembly of porous diblock copolymer films, Phys. Rev. E: Stat., Nonlinear, Soft Matter Phys., 2006, 73, 031608.

17 M. H. Nurmawati, P. K. Ajikumar, R. Renu and S. Valiyaveettil, Hierarchical self-organization of nanomaterials into two-dimensional arrays using functional polymer scaffold, Adv. Funct. Mater., 2008, 18, 3213-3218.

18 M. Srinivasarao, D. Collings, A. Philips and S. Patel, ThreeDimensionally Ordered Array of Air Bubbles in a Polymer Film, Science, 2001, 292, 79-83.

19 F. Pilati, M. Montecchi, P. Fabbri, A. Synytska, M. Messori, M. Toselli, K. Grundke and D. J. Pospiech, Design of surface properties of PET films: Effect of fluorinated block copolymers, J. Colloid Interface Sci., 2007, 315, 210-222.

20 W. Sun, J. Ji and J. Shen, Rings of nanoparticle-decorated honeycomb structured polymeric film: the combination of pickering emulsions and capillary flow in the breath figures method, Langmuir, 2008, 24, 11338-11344.

21 B. P. Nair and C. Pavithran, Micropatterned Surfaces through Moisture-Induced Phase-Separation of Polystyrene-Clay Nanocomposite Particles, Langmuir, 2010, 26(15), 12948-12952.

22 K. Kon, C. N. Brauer, K. Hidaka, H.-G. Lohmannsroben and O. Karthaus, Preparation of Patterned Zinc Oxide Films by Breath Figure Templating, Langmuir, 2010, 26(14), 12173-12176.

23 W. Sun, Z. Shao and J. Ji, Particle-assisted fabrication of honeycomb-structured hybrid films via breath figures method, Polymer, 2010, 51, 4169-4175.

24 V. Vohra, A. Bolognesi, G. Calzaferri and C. Botta, Multilevel organization in hybrid thin films for optoelectronic applications, Langmuir, 2009, 25, 12019-12023.

25 X. Jiang, X. Zhou, Y. Zhang, T. Zhang, Z. Guo and N. Gu, Interfacial Effects of in-Situ Synthesized Ag Nanoparticles on Breath Figures, Langmuir, 2010, 26(4), 2477-2483.

26 X. Li, L. Zhang, Y. Wang, X. Yang, N. Zhao, X. Zhang and J. Xu, A Bottom-Ip Appraoch to Fabricate Patterned Surfaces with Asymmetrical $\mathrm{TiO}_{2}$ microparticles trapped in the holes of honeycomb like Polymer Film, J. Am. Chem. Soc., 2011, 133, 3736-3739.

27 L.-S. Wan, Q.-L. Li, P.-C. Chen and Z.-K. Xu, Patterned biocatalytic films via one-step self-assembly, Chem. Commun., 2012, 48, 4417-4419.

28 H. Ma, J. Cui, A. Song and J. Hao, Fabrication of freestanding honeycomb films with through-pore structures via air/water interfacial self-assembly, Chem. Commun., 2011, 47, 1154-1156.

29 Y. Zhang and C. Wang, Micropatterning of proteins on 3D porous polymer film fabricated by using the breath-figure method, Adv. Mater., 2007, 19, 913-916.

30 E. Min, K. H. Wong and M. H. Stenzel, Microwells with patterned proteins by a self-assembly process using honeycomb-structured porous films, Adv. Mater., 2008, 20, 3550-3556.

31 B. B. Ke, L. S. Wan, P. C. Chen, L. Y. Zhang and Z. K. Xu, Tunable assembly of nanoparticles on patterned porous film, Langmuir, 2010, 26, 15982-15988.

32 L. S. Wan, J. Lv, B. B. Ke and Z. K. Xu, Facilitated and sitespecific assembly of functional polystyrene microspheres on patterned porous films, ACS Appl. Mater. Interfaces, 2010, 2, 3759-3765.

33 B. B. Ke, L. S. Wan, Y. Li, M. Y. Xu and Z. K. Xu, Selective layer-by-layer self-assembly on patterned porous films modulated by Cassie-Wenzel transition, Phys. Chem. Chem. Phys., 2011, 13, 4881-4887.

34 G. H. Tadayyon, S. M. Zebarjad and S. A. Sajjadi, Effect of both nano-size alumina particles and severe deformation on polyethylene crystallinity index, J. Thermoplast. Compos. Mater., 2011, 25(4), 479-490.

35 E. Ferrari, P. Fabbri and F. Pilati, Solvent and Substrate Contributions to the Formation of Breath Figure Patterns in Polystyrene Films, Langmuir, 2011, 27(5), 1874-1881.

36 B. Zhao, J. Zhang, X. Wang and C. Li, Water-assisted fabrication of honeycomb structure porous film from poly(L-lactide), J. Mater. Chem., 2006, 16, 509-513.

37 J. Peng, Y. Han, Y. Yang and B. Li, The influencing factors on the macroporous formation in polymer films by water droplet templating, Polymer, 2004, 45, 47-452.

38 Z. Guo, T. Pereira, O. Choi, Y. Wang and H. Thomas Hahn, Surface functionalized alumina nanoparticle filled polymeric nanocomposites with enhanced mechanical properties, J. Mater. Chem., 2006, 16, 2800-2808.

39 S. S. Babu, S. Mahesh, K. K. Kartha and A. Ajayaghosh, Solvent-Directed Self-Assembly of $\pi$ Gelators to Hierarchical Macroporous Structures and Aligned Fiber Bundles, Chem. Asian J., 2009, 4, 824-829. 